\title{
RISS Stage III Plasma Cell Myeloma
}

National Cancer Institute

\section{Source}

National Cancer Institute. RISS Stage III Plasma Cell Myeloma. NCI Thesaurus. Code C141396.

Serum beta-2-microg lobulin $5.5 \mathrm{mg} / \mathrm{L}$ or more and high-risk cytogenetics and/or high LDH. High risk cytogenetics consist of one or more of the following: del17p, $t(4 ; 14)$, or $\mathrm{t}(14 ; 16)$. (from AJCC 8th Ed.) 